\title{
The Development of the Greek Personality Assessment Inventory
}

\author{
Dimitrios Georgiou Lyrakos \\ Maastricht University, Elpis Care, Greece. \\ Email: info@lyrakos.gr \\ Received August 17 ${ }^{\text {th }}$, 2011; revised September 26 ${ }^{\text {th }}$, 2011; accepted October $25^{\text {th }}, 2011$.
}

\begin{abstract}
Presented in the current report are the reliability analysis of the Greek Personality Assessment Inventory (PAI), the differences between the means and the standard deviation (SD) of the Greek and the US sample and the creation of the maximum non-clinical T Scores for each of the scale and subscale produced by the Greek standardization sample. The PAI is considered, in the US and in many European countries, one of the most reliable multidimensional psychometric inventories. The PAI consists of 344 questions that are separated in non-overlapping scales of clinical, treatment focus, personality and validity context. The present, mixed design study had 1870 participants, males and females between the ages of 20 and 55.The participants were 1120 non-clinical (standardization sample), 450 psychiatric inpatients and 300 psychiatric outpatients, who were gathered from 4 major regions of Greece.
\end{abstract}

Keywords: Personality Assessment Inventory, Psychometrics, Greek Personality Tests, Clinical Psychology, Psychopathology, Clinical Assessment

\section{Introduction}

In the field of measures of personality there are a wide variety of instruments that can help to provide information that suggest either a diagnosis or diagnostic possibility. Even though these tests are not specifically designed to conclude an exact diagnosis, they nonetheless help to assist in the differential diagnosis with many complicated cases. These batteries include multi-scale instruments that list the symptoms and the other features of psychiatric disorders by asking the respondents to list how and in what capacity they are bothered by their symptoms. Respondents may be required to answer True or False as to whether particular statements apply to them. Research on these scales has indicated a high score on the individual level, or some patterns and profiles of multiple scores, to be associated with certain disorders or diagnostic groups. When presented, these scores are indicative of the presence of an associated type of pathology that needs further investigation. Hence the information provided by these testing forms is the starting point for the beginning of the diagnostic process. It also serves as supplemental information that helps to support an already suspected and present problem, information about possible treatment obstacles, or treatment focus areas and personality characteristics (Weiner et al., 2003).

One of the best known and most used among these measures is The Personality Assessment Inventory, (PAI) (Morey, 1991) which is a self-administered test for personality, psychopathology and treatment consideration factors. The test is designed to collect information on critical client variables in a number of behavioral health care situations. The test has been conceived to provide a measure of construct that prove to be central in the planning, implementation, and evaluation of treatment. Even though this instrument has been introduced very recently it has already been the source of considerable attention among researchers and clinicians. Additionally the PAI has been described as a 'substantial improvement from a psychometric perspective over the existing new personality tests' (Helmes et al., 1993). The PAI has been developed along the lines of a construct validation framework that emphasizes a rational and quantitative method of scale development which lays a strong emphasis on a theoretically informed approach towards developing items. In addition to this it also emphasizes an assessment of the items' stability and correlates. The PAI has been designed for use with individuals between the ages of 18 to 89 years of age and consists of 344 items constituting on four sets of nonoverlapping scales. There are 4 validity scales, 11 clinical scales covering major categories of pathologies that correspond to DSM nosology (neurotic spectrum—SOM, ANX, ARD, DEP; psychotic spectrum-MAN, PAR, SCZ; behavior disorder or impulse control problems-BOR, ANT, ALC, DRG). There are 5 treatment scales measuring constructs that are relevant to treatment, and 2 interpersonal scales (Strauss et al. 2006), on Table 1 there are presented the scales and subscales and their acronyms.

When Morey in 1991 was developing the PAI he reviewed each historical and recent literature on the targeted clinical syndromes in order to ensure that the PAI item assessed the core components of each disorder. For this reason the PAI has documented reliability validity in the assessment of personality and psychopathology in college, normative, and clinical sample. The PAI has also been examined recently in other specific populations such as alcohol dependent individuals, people with deviant and criminal behavior, as well as individuals suffering from eating disorders (Boccaccini et al. 2010, Sinclair et al. 2010, Thompson et al. 2010, Walters et al. 2010). The studies done with these groups indicate a success of PAI diagnosing these populations. There are certain aspects of PAI that indicate that it may be especially well suited for use with patients suffering from chronic pain as well (Karlin et al., 2005). A major practical advantage of PAI is its low text complexity of a fourth grade level of education. It is also much shorter than other personality inventories such as the MMPI-2 which has 567 items compared to the significant less questions on the PAI (Weiner et al., 2003, Lanyon et al. 1997). Additionally the PAI also contains a greater coverage of certain phenomena related to psychopathology and personality, including acting out, substance abuse and drugs treatment acceptance, interpersonal factors, and the more severe personality dysfunction such as 
Table 1.

Scales and subscales names and acronyms.

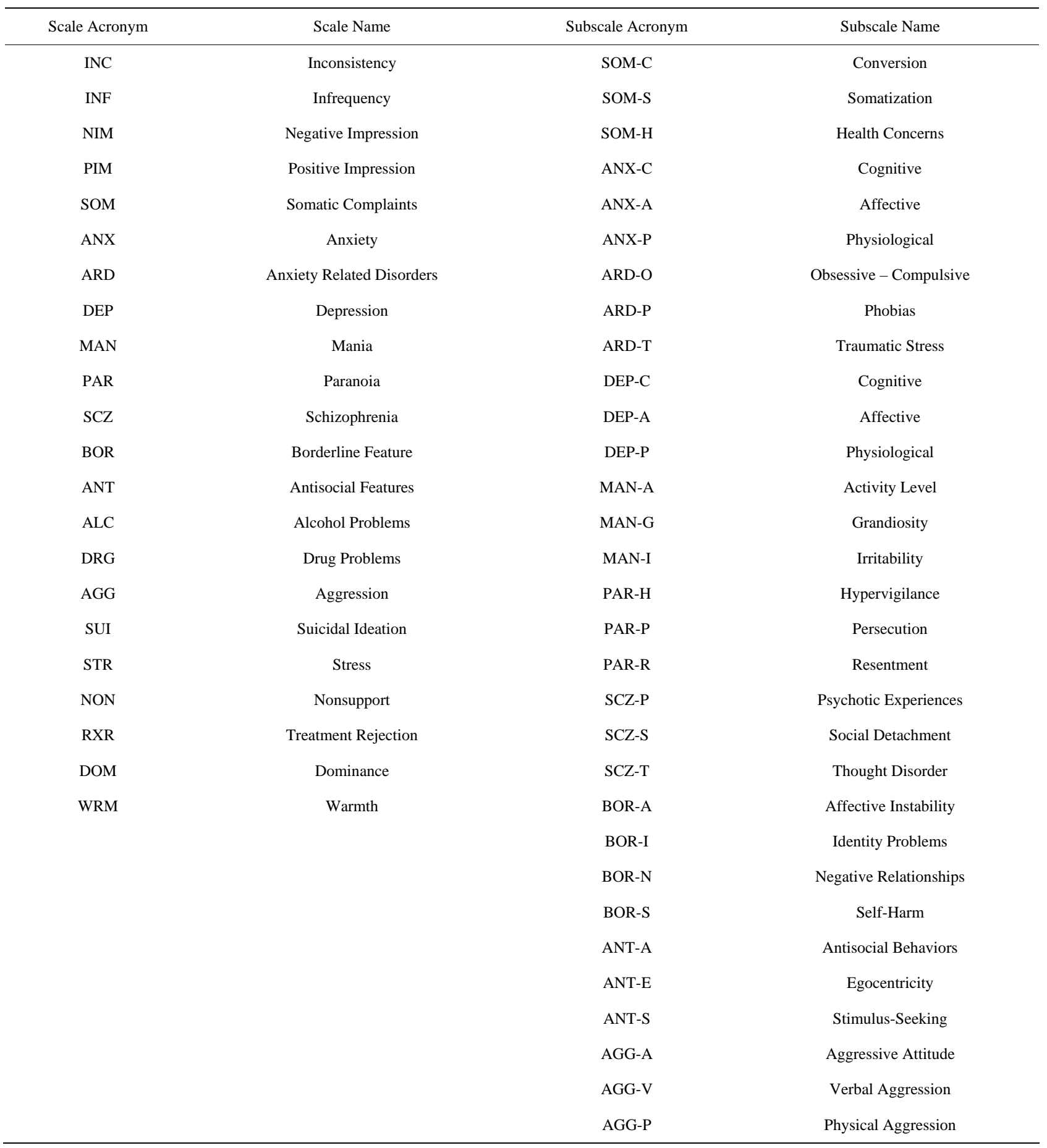

borderline personality and psychopathy (Weiner et al., 2003). The PAI also has an advantage over the other briefer psychological measures whereby it includes 5 main validity scales, infrequency, Inconsistency, positive, negative impression and malingering, which are particularly valuable patients in crisis, where they over describe their symptoms or they tend to conceal them (Hall, 2007).

The purpose of the present study is to demonstrate the development of the Greek Personality Assessment Inventory, based on the German study by Groves et al. 2007, and the
Greek T-Scores produced by the analysis of the standardization sample.

\section{Method}

\section{Design}

A mixed design has been followed. The independent variables are the Greek version of the PAI Questionnaire and the US version of the same questionnaire and the dependant vari- 
ables are the data from the standardization and clinical US sample obtained by Morey in 1991 during the development of the PAI and the data from the Standardization and clinical Greek sample, obtained for the present study.

\section{Participants}

The 1870 participants were both male (48.9\%) and female (51.1\%). The nonclinical sample consisted of 1.120 participants (59.8\%) and were selected by a marketing research company from the major Greek cities. There were also 450 inpatients (24\%) and 300 outpatients (16.2\%), who were selected by psychiatric institutions and private practices from ma same cities that the non clinical sample was selected. The participants were adults, between the ages of 18 and 75; possible organic symptoms such as dementia and mental retardation (IQ < 80) excluded the participant from the study. The possible exclusion of any participant occurred by the screening questions before the completion of the questionnaire.

There were 4 major Greek regions, selected, from where the all participants were obtained (Table 2). On the Greek regions the islands were not included because there were significant procedural problems.

\section{Nonclinical Participants (Standardization Sample)}

The nonclinical, standardization, participants were obtained by a marketing research company, through door to door requests, in order to obtain a sufficient sample in terms of geographical and demographical characteristics, similar method was used in the German translation by Groves et al. 2007. The possible participants were requested if they were willing to participate in the study. If they wished to do so, they signed the consent form. On the form there was an option for the participants, if they wished, to be informed about the results of the questionnaire.

\section{Clinical Participants}

For the inpatients participating in the study initial permission was requested by the scientific directors of the facilities.

The clinical participants were initially screened, by their therapists, in terms of intelligence and organic disorders. Furthermore the participants should have an initial or final diagnosis of any Axis I or II mental disorder by a clinical service. The participant's therapists, for inpatients and outpatients, were requested to brief them about the study and then to ask them if

Table 2.

Region's population and participant's percent.

\begin{tabular}{ccc}
\hline Name of the City & Actual Population & Sample Percent \\
\hline Thessaloniki & $1,057,825$ & 24.7 \\
Bolos-Larisa & $82,439+279,305=361,744$ & 8.46 \\
Athens & $2,664,776$ & 62.3 \\
Patra-Tripoli & $163,446+28,976=192,442$ & 4.5 \\
& $4,276,787$ & 100 \\
\hline
\end{tabular}

Based on Population Report of 2004. they were willing to participate. Before the completion of the questionnaire the participants were asked to sign a consent form.

\section{Materials}

For the present study there were no other tests used apart from the Greek version of the PAI. There was though a structured interview for the standardization sample in order to determine a number of factors, such as whether they are or were on any psychiatric medications, whether they ever visited a psychologist or psychiatrists and way, whether they have any alcohol or drug abuse problems. On the interview form there were also a number of clarification questions if the participant answered affirmatively to any of the above-mentioned questions.

\section{Procedure}

Before the Greek version of the PAI could be distributed, a translation and back translation of the English version should be approved by Les Morey, the writer of the original questionnaire. The translation was conducted by two different Greek, English teachers and then both translations were compared by two clinical psychologists. In order to evaluate the cross cultural validity of the questions a cultural psychologist had evaluated the questions. After the formulation of the Greek version of the questionnaire two different English teachers were requested to translate the Greek version of the questionnaire in English. After the approval of the back translation the distribution commenced. After obtaining approval by the scientific directors of the mental health services, we have provided to the facilities and therapists, questionnaires and the participant's screening parameters. The same materials and the interview form were also given to the marketing company for obtaining the standardization sample.

\section{Analysis}

The statistical analysis of the present study, was conducted with SPSS 17, and it is separated in three different parts:

1) Reliability for all three groups of participants, nonclinical, inpatients and outpatients;

2) Comparison of the means and the SD between the Standardization US and Greek sample and, if the means and SD differ

3) The calculation of the T Scores based on the same equation used in the original US PAI, but with the means and standard deviation produced by the Greek population.

\section{Results}

From Tables 3 and 4, it can be seen that all the scales and subscales have produced high Cronbach's Alpha in all three types of participants, healthy, inpatients, and outpatients. From Table 3 it can be seen that the reliability analysis for all the subscales in all three samples, health participants, inpatients, outpatients, has produced high Cronbach's Alpha. For the healthy sample the lower alpha produced was for the ARD-O $=0.653$, but even if any of the items were deleted there would be no significant change. For the inpatients the lower Cronbach's Alpha was produced for MAN-A $=0.683$, but again there would be no significant change if any of the items were deleted. Finally for the outpatients sample, the lowest Cronbach's Alpha was produced for SOM-C $=0.721$, which would not have any significant change if any of the items were deleted. From Table 4, it 
Table 3.

Subscales reliability analysis.

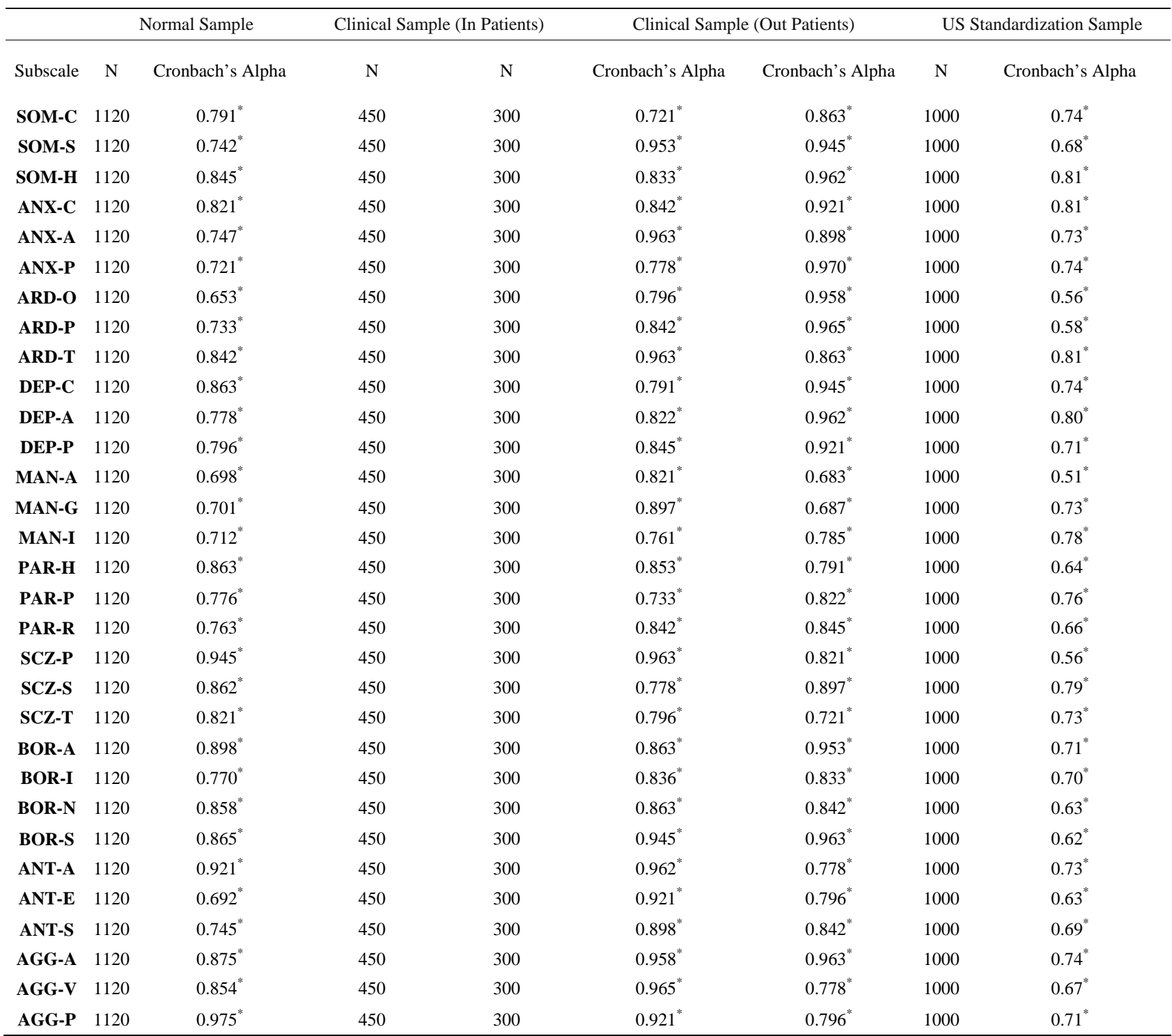

Note: ${ }^{*}$ No significant change of the Cronbach's Alpha if any of the items of the subscale are deleted.

can be seen that all the scales have produced high Cronbach's Alpha in all three types of participants, healthy, inpatients, and outpatients. The comparison of the alpha coefficients between the US (Morey 1991) and the Greek standardization sample do not present significant differences with the exemption of the ARD-O, ARD-P, MAN-A, SCZ-P and BOR-S where the reliability was significant higher in the Greek than in the US sample.

For the second part of the analysis the means and standard deviations have been compared between the standardization US sample (Morey 1991) and the Greek standardization sample produced in the present study, for the main scales and the subscales.

The comparison of the US and GR standardization Means (Table 5) showed that although the majority of the scales had no significant differences, there are scales with high mean differences between the US and Greek standardization sample presented below. The results presented are from the equation
US Mean-GR Mean.

$$
\begin{aligned}
\mathrm{SOM}=1.95, \mathrm{MAN}= & -2.1, \mathrm{PAR}=-2.8, \mathrm{BOR}=-5.15 \text { and } \\
& \mathrm{DOM}=1.49 .
\end{aligned}
$$

The comparison of the US and GR standardization SD (Table 5), produced high differences, with the exception of the PIM and NON scale. On Table 5 there are presented the Mean and SD of the US Normative sample (Morey 1991) and the obtained Greek Sample for the scales. The comparison of the Mean and the SD of US and GR Standardization sample of the subscales, based on the same equation, produced the highest differences for the means on: ARD-T $=-1.69$, DEP-C $=-1.23$, MAN-G $=-1.29$, BOR-A $=-1.36$ and BOR-I $=-1.2$. In terms of the SD, all subscales had similar differences, which is also consistent with previous findings by Karaminas et al. (2007), when they validated the MMPI-II.

As we have described, in the third phase of the analysis we have created T Scores based on the Greek standardization sample according to the original study of the PAI. The reason for 
Table 4.

Scales reliability analysis.

\begin{tabular}{|c|c|c|c|c|c|c|c|c|}
\hline \multirow[b]{2}{*}{ Subscale } & \multicolumn{2}{|r|}{ Normal Sample } & \multicolumn{2}{|c|}{ Clinical Sample (In Patients) (Outpatients) } & \multicolumn{2}{|c|}{ Clinical Sample (Out Patients) } & \multicolumn{2}{|c|}{ US Standardization Sample } \\
\hline & e $\mathrm{N}$ & Cronbach's Alpha & $\mathrm{N}$ & $\mathrm{N}$ & Cronbach’s Alpha & Cronbach’s Alpha & $\mathrm{N}$ & Cronbach’s Alpha \\
\hline NIM & 1120 & $0.852^{*}$ & 450 & 300 & $0.864^{*}$ & $0.967^{*}$ & 1000 & $0.72^{*}$ \\
\hline PIM & 1120 & $0.862^{*}$ & 450 & 300 & $0.943^{*}$ & $0.987^{*}$ & 1000 & $0.71^{*}$ \\
\hline SOM & 1120 & $0.792^{*}$ & 450 & 300 & $0.899^{*}$ & $0.898^{*}$ & 1000 & $0.89^{*}$ \\
\hline ANX & 1120 & $0.821^{*}$ & 450 & 300 & 0.778 & $0.979^{*}$ & 1000 & $0.90^{*}$ \\
\hline ARD & 1120 & $0.895^{*}$ & 450 & 300 & 0.796 & $0.983^{*}$ & 1000 & $0.76^{*}$ \\
\hline DEP & 1120 & $0.795^{*}$ & 450 & 300 & $0.799^{*}$ & $0.960^{*}$ & 1000 & $0.87^{*}$ \\
\hline MAN & 1120 & $0.954^{*}$ & 450 & 300 & $0.836^{*}$ & $0.861^{*}$ & 1000 & $0.82^{*}$ \\
\hline PAR & 1120 & $0.835^{*}$ & 450 & 300 & $0.821^{*}$ & $0.959^{*}$ & 1000 & $0.85^{*}$ \\
\hline SCZ & 1120 & $0.821^{*}$ & 450 & 300 & $0.894^{*}$ & $0.967^{*}$ & 1000 & $0.81^{*}$ \\
\hline BOR & 1120 & $0.742^{*}$ & 450 & 300 & $0.890^{*}$ & $0.987^{*}$ & 1000 & $0.87^{*}$ \\
\hline ANT & 1120 & $0.850^{*}$ & 450 & 300 & $0.864^{*}$ & $0.759^{*}$ & 1000 & $0.84^{*}$ \\
\hline ALC & 1120 & $0.865^{*}$ & 450 & 300 & $0.861^{*}$ & $0.845^{*}$ & 1000 & $0.84^{*}$ \\
\hline DRG & 1120 & $0.799^{*}$ & 450 & 300 & $0.959^{*}$ & $0.873^{*}$ & 1000 & $0.74^{*}$ \\
\hline AGG & 1120 & $0.833^{*}$ & 450 & 300 & $0.967^{*}$ & $0.799^{*}$ & 1000 & $0.85^{*}$ \\
\hline SUI & 1120 & $0.915^{*}$ & 450 & 300 & $0.861^{*}$ & $0.836^{*}$ & 1000 & $0.85^{*}$ \\
\hline STR & 1120 & $0.741^{*}$ & 450 & 300 & $0.959^{*}$ & $0.821^{*}$ & 1000 & $0.76^{*}$ \\
\hline NON & 1120 & $0.864^{*}$ & 450 & 300 & $0.967^{*}$ & $0.894^{*}$ & 1000 & $0.72^{*}$ \\
\hline RXR & 1120 & $0.843^{*}$ & 450 & 300 & $0.987^{*}$ & $0.890^{*}$ & 1000 & $0.76^{*}$ \\
\hline DOM & 1120 & $0.829^{*}$ & 450 & 300 & $0.759^{*}$ & $0.864^{*}$ & 1000 & $0.78^{*}$ \\
\hline WRM & 1120 & $0.832^{*}$ & 450 & 300 & $0.845^{*}$ & $0.987^{*}$ & 1000 & $0.79^{*}$ \\
\hline
\end{tabular}

Note: *No significant change of the Cronbach's Alpha if any of the items of the scale are deleted.

that is because, the Mean and SD of the Standardization sample is very important in the equation for the $\mathrm{T}$ Score, as it was demonstrated in the German Adaptation and validation by Groves et al. (2007). That is why it was decided to reevaluate all T Scores following the Example of Cheung et al. 2003 for the Chinese version of the PAI. In the present study we are presenting the maximum Nonclinical T Score.

From the Raw data produced by the Greek Standardization Sample the maximum raw scores were selected that was produced by $15 \%$ of the Healthy Population based on the Bell curve theory was used. With that selected raw scores and the Morey equation for the creation of the T Score (Morey, 1991), the Greek $\mathrm{T}$ scores were produced and they are presented on Table 6 for the scales and subscales.

\section{Discussion}

The aim of the present study was to validate the Greek PAI, taking into account the norms and social differences that Greece has in relation to the US, where the original PAI was developed. Although the majority of the PAI subscale T scores are not markedly different for the US, as they have been found by Morey 1991, and the Greek population, those differences can be substantial enough to produce or to hide a number of parameters important in treatment or in the process of producing a profile for a patient.

There were, however, three subscales and two scales that had high differences between the maximum normal US T score as found by Morey in 1991 and Greek T score, the three subscales are part of clinical scales, one scale is part of a treatment consideration section and the other scale examines personality traits.
The first subscale is the BOR-A which examines the shift of emotions. The Greek T score is 8 points higher in comparison to the US T score. In a study by Lolis (2004), it has been found that Greek people have a tendency to have outbursts of emotions more common than in Northern European and US people. BOR-A is a subscale that examines those behaviors and the participant's tendency towards them. The second subscale that produced a high difference between US and Greek T scores is BOR-N, which is the subscale that examines the tendency to focus on chaotic and problematic relationships. In this subscale the high nonclinical T-score is 10 points lower in comparison to the US, found by Morey (1991). This is consistent with the findings by Lolis (2004), who found that Greece has a stronger family setting and different standards in comparison to the US society (Lolis, 2004). Greeks have a tendency to rely more on friends and family and they also tend to expect more by their social surrounding (Lolis, 2004), if those expectations are not fulfilled then the person has a tendency to be disappointed and to consider his/her social network as insufficient and inadequate, exactly what the treatment scale of Nonsupport examines, which is the only scale with the US maximum nonclinical T score been 16 points higher in comparison to the Greek. That shows that the US standardization sample has a significant higher 'tolerance' in comparison to the Greek standardization factor in terms of disappointment by the participant's social network. The last subscale that produced significant different $\mathrm{T}$ score in Greece in comparison to the US is the ANT-E, which examines the empathy and interaction with others. Based on the results described above for NON and BOR-N, this 6 point difference is explainable, the lower $\mathrm{T}$ score in that subscale signifies a lower tolerance in the Greek community about acts of 'egocentricity'. In order to ensure though the accuracy of that 
Table 5 .

Means standard deviation US and Greek sample.

\begin{tabular}{|c|c|c|c|c|c|c|c|c|c|}
\hline Scale & US Mean & US SD & GR Mean & GR SD & Scale & US Mean & US SD & GR Mean & GR SD \\
\hline ICN & 5.39 & 3.35 & 5.5 & 3.45 & SOM-C & 2.5 & 3.37 & 2.34 & 2.99 \\
\hline INF & 2.66 & 2.57 & 3.46 & 2.77 & SOM-S & 4.51 & 3.73 & 4.33 & 3.54 \\
\hline NIM & 1.69 & 2.7 & 1.39 & 2.9 & SOM-H & 4.09 & 4.27 & 3.57 & 4.53 \\
\hline PIM & 15.07 & 4.36 & 14.86 & 4.66 & ANX-C & 6.05 & 4.33 & 6.37 & 4.132 \\
\hline ANX & 16.47 & 10.56 & 16.97 & 10.49 & ANX-P & 4.17 & 3.55 & 4.39 & 3.37 \\
\hline ARD & 19.91 & 8.3 & 20.2 & 8.5 & ARD-O & 9.33 & 3.75 & 9.14 & 3.817 \\
\hline DEP & 14.28 & 9.43 & 14.53 & 9.49 & ARD-P & 6.7 & 3.61 & 6.57 & 3.785 \\
\hline MAN & 23.01 & 9.22 & 25.11 & 10.32 & ARD-T & 3.88 & 4.12 & 4.17 & 3.92 \\
\hline PAR & 18.45 & 8.69 & 18.25 & 8.72 & DEP-C & 4.34 & 3.43 & 4.57 & 3.356 \\
\hline SCZ & 13.99 & 7.79 & 14.29 & 7.89 & DEP-A & 4.04 & 3.66 & 4.34 & 3.585 \\
\hline BOR & 18.03 & 10 & 18.18 & 9.89 & DEP-P & 5.89 & 4.16 & 5.58 & 3.742 \\
\hline ANT & 13.16 & 9.11 & 13.75 & 9.17 & MAN-A & 6.69 & 3.2 & 7 & 3.662 \\
\hline ALC & 4.83 & 5.62 & 4.57 & 5.58 & MAN-G & 8.39 & 4.37 & 9.68 & 4.99 \\
\hline DRG & 4.09 & 4.99 & 4.21 & 5.01 & MAN-I & 7.92 & 4.37 & 8.61 & 5.19 \\
\hline AGG & 14.81 & 8.42 & 15.86 & 8.5 & PAR-H & 7.6 & 4.27 & 7.93 & 3.88 \\
\hline SUI & 3.28 & 4.86 & 3.07 & 4.81 & PAR-P & 3.64 & 3.41 & 3.21 & 3.315 \\
\hline STR & 5.8 & 4.45 & 5.95 & 4.5 & PAR-R & 7.21 & 3.53 & 7.8 & 3.394 \\
\hline NON & 4.9 & 3.36 & 5.18 & 3.32 & SCZ-P & 4.09 & 2.99 & 4.11 & 3.125 \\
\hline RXR & 13.76 & 4.65 & 14 & 4.67 & SCZ-S & 5.59 & 3.94 & 5.26 & 3.716 \\
\hline \multirow[t]{10}{*}{ WRM } & 23.58 & 23.58 & 23.48 & 23.6 & BOR-A & 4.71 & 3.27 & 5.07 & 2.981 \\
\hline & & & & & BOR-I & 4.87 & 3.37 & 5.07 & 3.31 \\
\hline & & & & & BOR-N & 5.14 & 3.17 & 5.21 & 2.959 \\
\hline & & & & & BOR-S & 3.32 & 2.57 & 3.25 & 2.661 \\
\hline & & & & & ANT-A & 4.99 & 4.42 & 4.64 & 4.387 \\
\hline & & & & & ANT-E & 3.43 & 3.01 & 3.64 & 2.987 \\
\hline & & & & & ANT-S & 4.74 & 3.66 & 4.96 & 3.809 \\
\hline & & & & & AGG-A & 5.8 & 3.59 & 5.64 & 3.311 \\
\hline & & & & & AGG-V & 6.72 & 3.52 & 7.36 & 3.278 \\
\hline & & & & & AGG-P & 2.29 & 2.96 & 2.5 & 2.603 \\
\hline
\end{tabular}

US Sample N = 1000; Greek Sample N = 1380 .

subscale, interviews were conducted with participants from the standardization sample and from the clinical sample diagnosed with Antisocial Personality disorder, which supported the clinical threshold of T score 89 for the Greek clinical sample.

The last scale with significant difference between US and Greek maximum nonclinical T score is the personality scale of Warmth. That particular scale is a two-way evaluative scale (high and low), which means that a person with high levels on that particular scale tends to be characterized by exceptionally strong need to be accepted by others (Morey, 1991), whilst people with low scores on Warmth tend to be uneasy in social situations. People, who score low in that particular scale, pre- sent uneasiness in social interaction. Again, associated with all the above scales and subscales with differences between the US and Greek T scores, the lower nonclinical T score is 10 points lower in the Greek standardization sample in comparison to the US sample.

The PAI is considered one of the most valid and accurate instrument in the field of clinical psychology and diagnostic psychometrics (Maruish, 1999). The purpose of the present study was to create the Greek T scores, following the same procedure that Morey used in 1991 in the development of the PAI. It has been decided to follow that particular procedure not because the results produced by the instrument had weaknesses in the Greek 
Table 6.

Maximum nonclinical T-scores (scales subscales).

\begin{tabular}{|c|c|c|c|}
\hline Scale & Maximum Normal T Scores & Subscale & Maximum Normal T Scores \\
\hline ICN & 65 & SOM-C & 82 \\
\hline INF & 76 & SOM-S & 70 \\
\hline NIM & 74 & SOM-H & 94 \\
\hline PIM & 69 & ANX-C & 71 \\
\hline SOM & 85 & ANX-A & 70 \\
\hline ANX & 67 & ANX-P & 72 \\
\hline ARD & 70 & ARD-O & 65 \\
\hline DEP & 68 & ARD-P & 67 \\
\hline MAN & 73 & ARD-T & 79 \\
\hline PAR & 68 & DEP-C & 70 \\
\hline SCZ & 71 & DEP-A & 75 \\
\hline BOR & 77 & DEP-P & 71 \\
\hline ANT & 74 & MAN-A & 77 \\
\hline ALC & 69 & MAN-G & 75 \\
\hline DRG & 70 & MAN-I & 67 \\
\hline AGG & 73 & PAR-H & 65 \\
\hline SUI & 77 & PAR-P & 75 \\
\hline STR & 71 & PAR-R & 66 \\
\hline NON & 72 & SCZ-P & 79 \\
\hline $\mathrm{RXR}$ & 69 & SCZ-S & 75 \\
\hline DOM & 67 & SCZ-T & 72 \\
\hline \multirow[t]{10}{*}{ WRM } & 71 & BOR-A & 73 \\
\hline & & BOR-I & 71 \\
\hline & & BOR-N & 70 \\
\hline & & BOR-S & 75 \\
\hline & & ANT-A & 77 \\
\hline & & ANT-E & 89 \\
\hline & & ANT-S & 70 \\
\hline & & AGG-A & 67 \\
\hline & & AGG-V & 74 \\
\hline & & AGG-P & 70 \\
\hline
\end{tabular}

sample, but because we wanted to fully exploit the PAI's strengths for the Greek population, as it has been done for the Chinese version of the PAI (Cheung et al., 2003).Again, as it had been done by the US PAI team, we are currently in the process of comparing the US and Greek T scores, through interviews, clinical evaluations and a number of different clinical tests. The results from those analyses will be presented on future reports.

Apart from the finding presented in the present article there is an ongoing process to investigate possible correlations be- tween the scales and subscales of the Greek PAI and other personality inventories, such as MMPI-II and to create a more detailed analysis of the validity of the inventory in Greek.

\section{References}

Archer, R. P., Smith, S. R. (2008) Personality Assessment. Florida: CRC Press.

Boccaccini, M. T., Murrie, D. C., Hawes, S. W., Simpler, A., \& Johnson, J. (2010). Predicting recidivism with the personality assessment inventory in a sample of sex offenders screened for civil commitment as sexually violent predators. Psychological Assessment, 22, 142-148. doi:10.1037/a0017818

Cheung, F. M., Kwong, Y. Y., Zhang, J. (2003). Clinical validation of the chinese personality assessment inventory. Psychological Assessment, 25, 89-100. doi:10.1037/1040-3590.15.1.89

Groves, J. A., \& Engel, R. R. (2007). The German adaptation and standardization of the personality assessment inventory (PAI). Journal of Personality Assessment, 88, 49-56.

Hall, W. D., \& Mattick, R. P. (2007) Clinical update: Codeine maintenance is opioid dependence. The Lancet, 370, 550-552. doi:10.1016/S0140-6736(07)61273-2

Helmes, E., \& Reddon, J. R. (1993). A perspective on developments in assessing psychopathology: A critical review of the MMPI and MMPI-2. Psychological Bulletin, 113, 453-471. doi:10.1037/0033-2909.113.3.453

Karaminas, N., Georgakas, P., Kantas A., Tsaousis, I., Marini, K., Karakosta, A., Stathopoulos, K. (2007) Cross cultural robustness of MMPI-2: The Greek standardization. International Psychological Bulleting, 11, 24-26.

Karlin, B. E., Creech, S. K., Grimes, J. S., Clark, T. S., Meagher, M. W., \& Morey, L. C. (2005) The personality assessment inventory with chronic pain patients: psychometric properties and clinical utility. Journal of Clinical Psychology, 61, 1571-1585. doi:10.1002/jclp.20209

Lanyon, R. I., \& Goodstein, L. D. (1997) Personality Assessment. New Jersey: John Wiley and Sons.

Lolis, V. (2004) Social Differences between the Greek, the US and the British population. PhD Thesis, Athen: Pantion University, unpublished.

Maruish, M. E. (1999) The Use of Psychological Testing for Treatment Planning and Outcomes Assessment. New Jersey: Lawrence Erlbaum Associates.

Morey, L. C. (1991). The personality assessment inventory professional manual. Odessa, FL: Psychological Assessment Resources.

Sinclair, S. J., Antonius, D., Shiva, A., Siefert, C. J., Kehl-Fie, K., Lama, S., Blais, M. A. (2010). The psychometric properties of the Personality Assessment Inventory-Short Form (PAI-SF) in inpatient forensic and civil samples. Journal of Psychopathology and Behavioral Assessment, 32, 406-415. doi:10.1007/s10862-009-9165-X

Strauss, E., Sherman, E. M. S., Spreen, O. (2006) A compendium of neuropsychological tests: Administration, norms, and commentary. Oxford: Oxford University Press.

Thompson, A. W., Hantke, N., Phatak, V., \& Chaytor, N. (2010). The Personality Assessment Inventory as a tool for diagnosing psychogenic nonepileptic seizures. Epilepsia, 51, 161-164. doi:10.1111/j.1528-1167.2009.02151.x

Wagner, M. T., Wymer, J. H., Topping, K. B., Pritchard, P. B. (2005) use of the personality assessment inventory as an efficacious and cost-effective diagnostic tool for nonepileptic seizures. Epilepsy and Behavior, 7, 301-304. doi:10.1016/j.yebeh.2005.05.017

Walters, G. D., Diamond, P. M., \& Magaletta, P. R. (2010). What is the latent structure of alcohol use disorders? A taxometric analysis of the personality assessment inventory alcohol problems scale in male and female prison inmates. Psychology of Addictive Behaviors, 24, 26-37. doi:10.1037/a0016587

Weiner, I. B., Freedheim, D. K., Goldstein, A. M. (2003) handbook of psychology: forensic psychology. New Jersey: John Wiley and Sons.

Weiner, I. B., et al. (2003) Handbook of Psychology Assessment Psychology. New Jersey: John Wiley and Sons.

Greek National Statistical Department (2004) Greek Population Report. 